\title{
Multiple Access Game in Ad-hoc Network
}

\author{
Hamidou Tembine *, Eitan Altman ${ }^{+}$, Rachid El-Azouzi * and Yezekael Hayel * \\ * LIA-CERI, University of Avignon \\ 39, chemin des Meinajaries, Agroparc BP 1228, 84911 Avignon Cedex 9, France \\ \{hamidou.tembine,rachid.elazouzi,yezekael.hayel\}@univ-avignon.fr \\ + INRIA \\ 2004 Route des Lucioles, 06902 Sophia Antipolis, France. \\ eitan.altman@sophia.inria.fr
}

\begin{abstract}
We study a Modified Multiple Access Game (MMAG) in two approach: repeated game approach and evolutionary game approach. We compute Nash equilibria and Evolutionary Stable Strategy (ESS) in the two cases. We study the delay impact on the performance of the evolutionary MMAG describing competition between mobile terminals over the access to a channel. We discuss about the convergence to the ESS in replicator dynamics and imitate better dynamics with delays.
\end{abstract}

Keywords: multiple access game, imitation, delayed dynamics

\section{INTRODUCTION}

Game theory has been used in several areas of communication theory including flow control $[1,21]$ and routing $[4$, $9,26]$. One of the approach studied for the medium access control (MAC) is the non-cooperative approach of the classical game theory. Users are rational and maximize their own payoff by choosing their probability of transmission for each slot. Evolutionary game theory has been proposed by biologists for studying competitive behavior of population instead of finite number of users. Moreover, the notion of equilibrium, which is called an evolutionary stable strategy is stronger than the standard Nash equilibrium as the ESS describes robustness of the equilibrium to mutations in the population strategies. Our aim in this paper is to consider an evolutionary game approach for the competitive MAC problem. Moreover, we study the stability property, in the sense of population dynamics, for this non-cooperative game. The Aloha and the subsequent slotted-Aloha protocols were introduced to improve the utilization of the shared medium by allowing user to send their traffic given some probability of transmission. Because slotted-Aloha exhibits an instability as the number of nodes increases [25], early research focused

Permission to make digital or hard copies of all or part of this work for personal or classroom use is granted without fee provided that copies are not made or distributed for profit or commercial advantage and that copies bear this notice and the full citation on the first page. To copy otherwise, to republish, to post on servers or to redistribute to lists, requires prior specific permission and/or a fee.

GameComm '07, October 22, 2007, Nantes, France

Copyright 2007 ICST 978-963-9799-00-4. on stabilizing the Aloha protocols [15]. For example, in [22], authors discussed the stability of slotted-Aloha with selfish user behavior and perfect information. A non-cooperative game formulation as been proposed in [20].

In this paper, we study a multiple access game in which there is a large population of mobile terminals in ad hoc network with low density [32]. A mobile decide to transmit or not a packet to a receiver when they are within transmission range of each other. Interference occurs as in the ALOHA protocol: if more than one neighbors transmit a packet at the same time then there is a collision. Multiple Access Game introduces the problem of medium access (see [8]). We modify the game in [32] and study the multiple access game in the two following perspectives:

(i) a regret cost in the multiple access game when no users transmit and study the discounted repeated game equilibria,

(ii) an evolutionary perspective of this modified multiple access game in which we study delay impact on the convergence to the ESS in two dynamics:replicator dynamics and imitate better dynamics.

The regret cost describe the behavior of mobiles when they are aware of the risk of collision. Risk is an important factor to be taken into consideration while managing revenues. The risk-aware is used in many field areas as security [34], traffic engineering routing [23] and management in autonomous agents [24]. We show that when the regret cost is lower than unit, and the delay is large, the system is not stable in the replicator dynamics and the system oscillates in the imitate better dynamics.

To improve the system performance in ad hoc network, we study the effect of the regret cost for transmission probability. We show that this cost can improve the system performance of ad hoc networks and that an appropriate pricing can be chosen that yields an equilibrium performance that optimizes the system performance.

The paper is structured as follows. We first provide in the next section the (modified) multiple access game in noncooperative context. We then study the discounted repeated multiple access game in section 3. After that, we introduce evolutionary MMAG with replicator and imitate better dynamics in section 4 and we discuss about stability conditions and convergence to the ESS. We conclude by numerical investigation of these dynamics in section 5 .

\section{MODIFIED MUTIPLE ACCESS GAME}


We consider a large population of mobile terminals in ad hoc network with low density (see figure 1). We assume that the density of the network is low, so that if a terminal attempts transmission one can neglect the probability of interference from more than one other mobile (called "neighbor"). We assume the mobiles move frequently and they have a

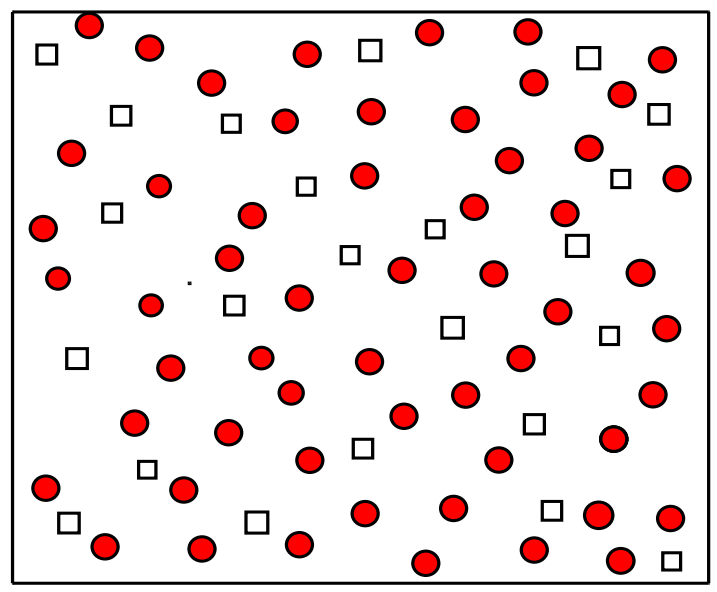

Figure 1: Population of mobiles in an Ad-Hoc Network. A square represents a destination, a circle represents a source.

packet to send in each time slot. Interference occurs as in the ALOHA protocol: if more than one neighbors transmit a packet at the same time then there is a collision. Multiple Access Game introduces the problem of medium access (see [8]). The Multiple Access Game is a symmetric nonzerosum game, the users have to share a common resource, the wireless medium, instead of providing it. Assume that the users use pure strategy. There are two users Player I and Player II who want to send some packets to their receivers R1 and R2 using a shared medium. Suppose furthermore that Player I, Player II, R1 and R2 are in the power range of each other, hence their transmissions mutually interfere. Each of the users has two possible strategies: either transmit $(T)$ or to stay quiet $(S)$. If Player $I$ transmits his packet, it incurs a transmission cost of $K \Delta \in(0,1)$ after a delay $\tau_{T}$. The packet transmission is successful if Player $I I$ does not transmit (stays quiet) in that given time slot and its delay is $\tau_{S}$, otherwise there is a collision. If there is no collision, Player I gets a reward of $K$ from the successful packet transmission after the delay $\tau_{T}$. When the two players stay quiet, they have a cost $\kappa K$. If $\kappa=0$ and $K=1$, we have the game described in [8]. The regret cost $\kappa$ describes the behavior of mobiles when they are aware of the risk of collision.

The interaction is represented in figure 2 .

\begin{tabular}{|c|c|c|c|}
\hline \multirow{3}{*}{ Player I receives } & & $T$ & $S$ \\
\hline & $T$ & $-\Delta K$ & $K-\Delta K$ \\
\hline & $S$ & 0 & $-\kappa K$ \\
\hline
\end{tabular}

Figure 2: Modified Multiple Access Game. $K$ is a positive parameter.

Nash Equilibria and Pareto optimality This matrix game has two pure Nash equilibria $(T, S)$ and $(S, T)$ and a unique mixed Nash equilibrium given by $\left(\frac{1-\Delta+\kappa}{1+\kappa}, \frac{\Delta}{1+\kappa}\right)$. Note that the pure strategies $(T, S)$ and $(S, T)$ are also optimal in the Pareto ${ }^{1}$ sense. The mixed equilibrium converges the pure strategy $T$ when the regret cost is large.

\section{DISCOUNTED REPEATED MMAG WITH FULL MONITORING}

We investigate the (infinitely) repeated game, i.e., the entire game represented by Fig.2 is played infinitely often. The repeated game is assumed to be discounted, i.e., the reward received at time $t$ is discounted by for some $\delta^{t}$. In general, there are many more Nash equilibria in the repeated game than simply repeating a stage game Nash equilibrium.

The model The same static game, called also the stage game, is played an infinite number of times. At the end of each stage, each player is aware of all the actions of all the players at times 1 through $t-1$. The overall payoff of player is the discounted sum of its payoff at each stage, for some discount factor $\delta \in(0,1)$, normalized by $1-\delta$.

Equilibria A subgame after a history $h_{t}$ is by itself a welldefined repeated game that starts at the time $t+1$. A Nash equilibrium is a subgame-perfect Nash equilibrium if for all time $t$ and for all history the players' strategies constitute a Nash equilibrium in every subgame induced by the history.

Let $m^{i}$ the maxmin point of the player $i$ in the stage game. Note by $I R$ the set of individually rational feasible payoff region i.e the portion of feasible region that Paretodominates the maxmin point. The set $I R$ is a full dimensional set. Thus, by the discounted folk theorem [13, 14, $31,10,11,7]$, the limit of the perfect public equilibria set when the users are patient $(\delta \rightarrow 1)$ is $I R$. Fudenberg, Levine and Takahashi[12] extended the folk theorem when the full dimensionally condition fails.

The maxmin point is given by $m^{1}=m^{2}=-K \frac{\Delta \kappa}{1+\kappa}$. The set $I R$ is represented in Fig.3.

\section{EVOLUTIONARY APPROACH}

Related works The evolutionary game framework is used for large populations that have many local interactions, each involving a small number of users. The ESS, first been defined in 1972 by the biologist M. Smith [30], is characterized by a property of robustness against invaders (mutations). More specifically, (i) if an ESS is reached, then the proportions of each population do not change in time. (ii) at ESS, the populations are immune from being invaded by other small populations. The conditions to be an ESS [27, 19] can be related to and interpreted in terms of Nash equilibrium in a matrix game. There is a close relation between the rest points of the replicator equation (more generally in payoff monotonic dynamics [36]) and the Nash equilibria of the related (symmetric) matrix game given by the so called folk theorem of evolutionary game theory $[5,18,36]$.

Although ESS has been defined in the context of biological systems, it is highly relevant to engineering as well (see [35]). Bonneau et al. have introduced evolutionary games in the context of unslotted ALOHA in [3]. The authors have identified conditions for the existence of non trivial ESS and

\footnotetext{
${ }^{1}$ An allocation of payoffs is said Pareto-optimal if the outcome cannot be improved upon without hurting at least one user.
} 


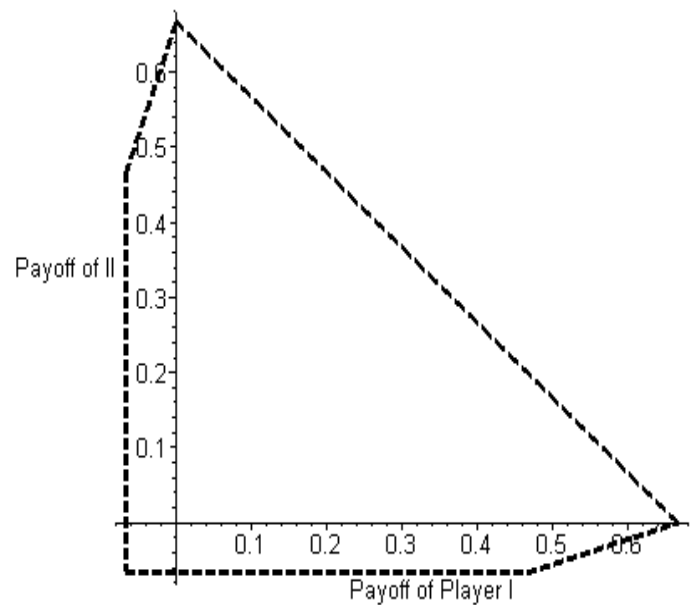

Figure 3: $I R$ of multiple access game. When $\kappa>0$ the perfect Nash equilibria payoff set is larger than the case $\kappa=0$.

have computed them explicitly for the ALOHA scheme. In [32], the authors consider the multiple access game without cost when the no users transmit and study delay effect in many evolutionary game dynamics with asymmetric delay [33].

\section{ESS}

We assume that the strategy $T$ has a delay $\tau$ and the strategy $S$ no delay. Let $\mathcal{S}:=\{T, S\}$ the set of strategies and $a_{k j}$ the fitness of subpopulation using $k$ against the subpopulation $j$.

The repartition of the cell can be represented by the probability $s$ to transmit. The number $s$ is the proportion of users in the cell using the strategy $T$. Then $s T+(1-s) S$ is the state of the cell. We denote this state by $(s, 1-s)$ or by the parameter $s$. Let $\Delta(\mathcal{S}):=\{(s, 1-s) \mid 0 \leq s \leq 1\}$ the set of mixed strategies.

Suppose that, initially, the population profile is $(s, 1-$ $s) \in \Delta(\mathcal{S})$. The average payoff in the population is $u(s, s)=$ $(s, 1-s) A\left(\begin{array}{c}s \\ 1-s\end{array}\right)$ where

$$
A=K\left(\begin{array}{cc}
-\Delta & 1-\Delta \\
0 & -\kappa
\end{array}\right)
$$

Now suppose that a small group of mutants enters this population playing according to a different profile (mut, $1-m u t)$. If we call $\epsilon \in(0,1)$ the size of the subpopulation of mutants after normalization, then the population profile after mutation will be $\epsilon$ mut $+(1-\epsilon) s$. After mutation, the average payoff of non-mutants who are randomly matched to mutants is given by

$$
u(s, m u t)=(s, 1-s) A\left(\begin{array}{c}
m u t \\
1-m u t
\end{array}\right),
$$

and the average payoff of non-mutants will be given by

$$
u(s, \epsilon \text { mut }+(1-\epsilon) s)=\epsilon u(s, m u t)+(1-\epsilon) u(s, s) .
$$

Analogously, we can construct the average payoff of mutant $u(m u t, \epsilon m u t+(1-\epsilon) s)$. A proportion of transmitters $s \in[0,1]$ is an ESS if for all $m u t \neq s$, there exists some $\epsilon_{m u t} \in(0,1)$, which may depend on mut, such that for all $\epsilon \in\left(0, \epsilon_{m u t}\right)$

$$
u(s, \epsilon m u t+(1-\epsilon) s)>u(m u t, \epsilon m u t+(1-\epsilon) s)
$$

That is, $s$ is ESS if, after mutation, non-mutants are more successful than mutants, in which case mutants cannot invade and will eventually get extinct. The number $\epsilon_{m u t}$ is called invasion barrier [36]. It is the maximum rate of mutants against which $s$ is resistant. If $s$ is an ESS then $s$ is a Nash equilibrium. Equivalently $s$ is an ESS if and only if it meets the best reply conditions:

$$
u(m u t, s) \leq u(s, s), \forall \text { mut },
$$

$u(m u t, s)=u(s, s) \Rightarrow u($ mut, $m u t)<u(s$, mut $) \quad \forall m u t \neq s$

We show that the mixed Nash equilibrium given Section 2 is an ESS. The state $\frac{1-\Delta+\kappa}{1+\kappa}$ (resp. $\left.\frac{\Delta}{1+\kappa}\right)$ represents proportion of individuals which transmit (resp. stay quiet). When the two subpopulation use this strategy, they obtain the same payoff equal $-K \frac{\Delta}{1+\kappa}$ which is negative. The strategy $\left(\frac{1-\Delta+\kappa}{1+\kappa}, \frac{\Delta}{1+\kappa}\right)$ is the unique interior Nash equilibrium. It is the unique symmetric Nash equilibrium. Thus, it is the only candidate to be ESS because symmetric Nash equilibria set contains ESS set. At a mixed equilibrium, the strategies $T$ and $S$ must have the same fitness. We check the condition of ESS given in (1). For all $\xi \neq \frac{1-\Delta+\kappa}{1+\kappa}$

$$
\begin{gathered}
\left(\frac{1-\Delta+\kappa}{1+\kappa}-\xi, \xi-\frac{(1-\Delta+\kappa)}{1+\kappa}\right) A\left(\begin{array}{c}
\xi \\
1-\xi
\end{array}\right) \\
=\frac{K}{1+\kappa}((1+\kappa) \xi-1+\Delta+\kappa)^{2}>0
\end{gathered}
$$

We conclude that $\left(\frac{1-\Delta+\kappa}{1+\kappa}, \frac{\Delta}{1+\kappa}\right)$ an ESS.

\section{Replicator Dynamics}

Replicator dynamics is one the most studied dynamics in evolutionary game theory. The replicator dynamics has been used for describing the evolution of road traffic congestion in which the fitness is determined by the strategies chosen by all drivers [28]. It has also been studied in the context of the association problem in wireless networks in [29]. We introduce the replicator dynamics which describes the evolution in the population of the various strategies. In the delayed replicator dynamics[33], the share of a strategy in the population grows at a rate equal to the difference between the delayed payoff of that strategy and the average delayed payoff of the population. If $(\xi(t), 1-\xi(t))$ denotes de repartition of the population in the multiple access game, then the fitness of the subpopulation using the strategy $T$ is $K(-\xi(t-\tau)+1-\Delta)$, and the fitness of the strategy $S$ is $-\kappa K(1-\xi(t))$. The replicator dynamic equation is given by

$\dot{\xi}(t)=-K \xi(t)(1-\xi(t))[\xi(t-\tau)+\kappa \xi(t)-1+\Delta-\kappa]$

Definitions 1. 1. The state $\xi^{*}$ is stationary state (or rest point, see [16]) of the delay differential equation (2) if it is a critical point i.e the right side of (2) is zero at $\xi^{*}$. This condition becomes $\xi^{*} \in\{0,1\}$ or $f_{1}(\xi)=$ $f_{2}(\xi)$

2. $\xi^{*}$ is stable if it is a stationary point with the property that for every neighborhood $\mathcal{V}$ of $\xi^{*}$, there exists a 
neighborhood $\mathcal{U} \subset \mathcal{V}$ with the property that if $\xi(t) \in \mathcal{U}$ for $t \in(-\tau, 0)$ then $\xi(t) \in \mathcal{U}$ for all $t>0$.

3. $\xi^{*}$ is asymptotically stable if it is stable and there exists a neighborhood $\mathcal{W}$ of $\xi^{*}$ such that $\xi(t) \in \mathcal{W}$ for all $t \in(-\tau, 0)$ implies $\lim _{t \rightarrow+\infty} \xi(t)=\xi^{*}$

It is known [2, pp.336],[17, pp.188] or from the Hartman and Grobman theorem adapted to delay differential equation that the steady state $\left(\frac{1-\Delta+\kappa}{1+\kappa}, \frac{\Delta}{1+\kappa}\right)$ is asymptotically stable for (2) around the stationary point $\frac{1-\Delta+\kappa}{1+\kappa}$ if the trivial solution of the linearized version is asymptotically stable.

The trivial solution of the linear delay differential equation

$$
\dot{z}(t)=-\frac{K \xi^{*}\left(1-\xi^{*}\right)}{1+\kappa}[\kappa z(t)+z(t-\tau)], \tau>0,
$$

is asymptotically stable if and only if all roots of the characteristic equation

$$
\lambda+\frac{K \xi^{*}\left(1-\xi^{*}\right)}{1+\kappa}\left[\kappa+e^{-\tau \lambda}\right]=0
$$

have negative real parts. If there exists a roots of (4) with positive real parts, the trivial solution is not asymptotically stable. Note that if $\lambda=\Re(\lambda)+i \Im(\lambda)$ is solution of the characteristic equation then its conjugate $\Re(\lambda)-i \Im(\lambda)$ is also solution. The difficulty about (4) is that it is transcendental equation and hence the determination of condition for stability is highly nontrivial.

The following Rouchés theorem on the continuity of the roots of an equation as a function of parameters will be needed in analyzing the characteristic equation (4). A proof of this theorem can be found in Dieudonné [6].

Theorem 1 (Rouché). Let $A$ be an open set in $\mathbb{C}$, the set of complex numbers, $F$ a metric space, $\phi$ a continuous complex valued function in $A \times F$, such that, for each $\alpha \in$ $F, z \longmapsto \phi(z, \alpha)$, is analytic in $A$. Let $B$ be an open set of $A$, whose closure $B$ in $\mathbb{C}$ is compact and contained in $A$, and let $\alpha_{0} \in F$ be such that no zero of $\phi(z, \alpha)$ is on the frontier of $B$. Then there exists a neighborhood $W$ of $\alpha_{0}$ in $F$ such that

- for any $\alpha \in W, \phi(z, \alpha)$ has no zeros on the frontier of $B$

- for any $\alpha \in W$, the sum of the orders of the zeros of $\phi(z, \alpha)$ belonging to $B$ is independent of $\alpha$

Proposition 1. If $\kappa \geq 1$ then all roots of the characteristic equation (4) has negative real parts.

Proof. - Suppose there exists root $\lambda$ of the characteristic equation (4) with $\Re(\lambda)>0$ and $\kappa>1$ then $\Re(\lambda)+K \xi^{*}\left(1-\xi^{*}\right) \frac{\kappa}{1+\kappa}>K \xi^{*}\left(1-\xi^{*}\right) \frac{\kappa}{1+\kappa}>\frac{K \xi^{*}\left(1-\xi^{*}\right)}{1+\kappa}>$ $-\frac{K \xi^{*}\left(1-\xi^{*}\right)}{1+\kappa} e^{-\tau \Re(\lambda)} \cos (\tau \Im(\lambda))$ a contradiction with (4). Thus all roots of the characteristic equation (4) have real parts strictly negative.

- Suppose that $\kappa=1$.

$$
\left\{\begin{array}{c}
\Re(\lambda)+K \xi^{*}\left(1-\xi^{*}\right) \frac{\kappa}{1+\kappa}= \\
=-K \xi^{*}\left(1-\xi^{*}\right) \frac{\kappa}{1+\kappa} e^{-\tau \Re(\lambda)} \cos (\tau \Im(\lambda)) \\
\Im(\lambda)=K \xi^{*}\left(1-\xi^{*}\right) \frac{\kappa}{1+\kappa} e^{-\tau \Re(\lambda)} \sin (\tau \Im(\lambda))
\end{array}\right.
$$

If $\Re(\lambda)>0$ then $\Re(\lambda)+K \xi^{*}\left(1-\xi^{*}\right) \frac{\kappa}{1+\kappa}=-K \xi^{*}(1-$ $\left.\xi^{*}\right) \frac{\kappa}{1+\kappa} e^{-\tau \Re(\lambda)} \cos (\tau \Im(\lambda)) \leq K \xi^{*}\left(1-\xi^{*}\right) \frac{\kappa}{1+\kappa}$ i.e $\Re(\lambda) \leq$ 0 : impossible. If $\Re(\lambda)=0$ then $\Im(\lambda)=0$. The trivial solution is stable.

Proposition 2. Suppose that $\kappa<1$. Then all of the characteristic equation (4) has negative real parts if and only if

$$
\tau \leq \frac{(1+\kappa)^{2}}{K \Delta(1-\Delta+\kappa)} \cos ^{-1}(-\kappa) \sqrt{\frac{1+\kappa}{1-\kappa}}
$$

Note that when $\kappa$ goes to 1 , the bound

$$
\frac{(1+\kappa)^{2}}{K \Delta(1-\Delta+\kappa)} \cos ^{-1}(-\kappa) \sqrt{\frac{1+\kappa}{1-\kappa}}
$$

goes to infinity (stability without conditions).

Proof. - Suppose that

$$
\tau<\tau_{0}:=\frac{(1+\kappa)^{2}}{K \Delta(1-\Delta+\kappa)} \cos ^{-1}(-\kappa) \sqrt{\frac{1+\kappa}{1-\kappa}} .
$$

When $\tau=0$ the system (4) become $\Im(\lambda)=0$ and $\Re(\lambda)=-K \frac{\Delta(1-\Delta+\kappa)}{(1+\kappa)^{2}}<0$.

Let $b=K \frac{\Delta(1-\Delta+\kappa)}{(1+\kappa)^{2}}, a=\kappa b$. If (4) has a solution with real part strictly positive, then there must be some $0<\tau_{1}<\tau_{0}$ such that (4) has purely imaginary roots $\pm \Im(\lambda) \times i$ with $\Im(\lambda)>0$ satisfying

$$
\begin{gathered}
\left\{\begin{array} { c } 
{ a = - b \operatorname { c o s } ( \tau _ { 1 } y ) } \\
{ y = b \operatorname { s i n } ( \tau _ { 1 } y ) }
\end{array} \Longleftrightarrow \left\{\begin{array}{c}
a=-b \cos \left(\tau_{1} y\right) \\
y=b \sin \left(\tau_{1} y\right), y>0
\end{array}\right.\right. \\
\Longleftrightarrow\left\{\begin{array}{c}
y=\sqrt{b^{2}-a^{2}} \\
\tau_{1}=\tau_{0}
\end{array}\right.
\end{gathered}
$$

We prove that $\tau_{1}=\tau_{0}$ which is a contradiction. Thus, if $0<\tau<\tau_{0}$ then all solutions of the characteristic equation (4) have negative real parts.

- Suppose that $\tau>\tau_{0}$.

Denote by $s=\lambda / b, r=\tau b>0, r_{0}=\tau_{0} b$. The equation (4) can be written as the zeros the function

$$
\phi(s, \kappa):=s+\kappa+e^{-r s}
$$

We want to prove that all zeros of $\phi(., \kappa)$ have negative real parts. The function $\phi$ is an analytic function in $s$ and $\kappa$. Let

$$
\mathcal{O}:=\{s, \Re(s) \geq 0,|s| \leq 1\}
$$

One has $r_{0} \geq \frac{\pi}{2}$. Indeed,

$$
\arccos (-p)-\frac{\pi}{2} \sqrt{1-p^{2}}>0, p \in(0,1) .
$$

Thus when $r>r_{0}$ and $r \neq 2 k \pi+\frac{\pi}{2}, k \in \mathbb{N}$ the function $\phi(s, 0)$ has no zeros in the boundary of $\mathcal{O}$. Thus Rouche's theorem 1 implies that there exists a $\delta>0$ such that $\kappa<\delta \Rightarrow \phi(s, 0)$ and $\phi(s, \kappa)$ have same sum of the orders of zeros. When $r>r_{0}$ the zeros of $\phi(s, 0)$ is at least 2 . Thus, $\phi(s, \kappa)$ has at least one root with positive real part. 


\section{Imitate Better Dynamics}

A delayed myopic imitation dynamic has been introduced in [32]. We suppose that users in the population review their strategy and imitate the better's strategy on the time (imitation by dissatisfaction). In a symmetric two player game, the delayed dynamic is given by

$$
\dot{x}_{k}(t)=x_{k}(t)\left(\sum_{j=1}^{n} x_{j}(t)\left[\rho_{k j}\left(x\left(t-\tau_{k}\right)\right)-\rho_{j k}\left(x\left(t-\tau_{j}\right)\right)\right]\right)
$$

where $\rho_{k j}(x)=g\left(f_{k}(x), f_{j}(x)\right), k, j=1,2$ and $g(a, b)=0$ if $a<b$ and $g(a, b)=1$ if $a>b$. This equation can be expressed as

$$
\dot{\xi}(t)=\xi(t)(1-\xi(t)) h(\xi(t-\tau), \xi(t))
$$

where

$h(\xi(t-\tau), \xi(t))=\left\{\begin{array}{cll}1 & \text { if } & \xi(t-\tau)+\kappa \xi(t)<1-\Delta+\kappa \\ 0 & \text { if } & \xi(t-\tau)+\kappa \xi(t)=1-\Delta+\kappa \\ -1 & \text { if } & \xi(t-\tau)+\kappa \xi(t)>1-\Delta+\kappa\end{array}\right.$

Proposition 3. Suppose that $\kappa<1$. For all $\tau>0$, and for all initial conditions $\forall t \in(-\tau, 0], \xi(t)=\xi_{0} \in$ $\left(0, \frac{1-\Delta+\kappa}{1+\kappa}\right)$ the solution of imitation equation oscillates around the ESS.

Proof. Consider the following differential equations

$$
\left\{\begin{array}{ccc}
\dot{\xi}(t)=\xi(t)(1-\xi(t)) & \text { if } & \xi(t-\tau)+\kappa \xi(t)<1-\Delta+\kappa \\
\dot{\xi}(t)=0 & \text { if } & \xi(t-\tau)+\kappa \xi(t)=1-\Delta+\kappa \\
\dot{\xi}(t)=-\xi(t)(1-\xi(t)) & \text { if } & \xi(t-\tau)+\kappa \xi(t)>1-\Delta+\kappa \\
\forall t \in(-\tau, 0], \xi(t)=\xi_{0} & &
\end{array}\right.
$$

$$
(* *) \quad\left\{\begin{array}{c}
\dot{w}(t)=w(t)(1-w(t)) \\
w(0)=\xi_{0}
\end{array}\right.
$$

where $\xi_{0} \in\left(0, \frac{1-\Delta+\kappa}{1+\kappa}\right)$.

The solution of $(* *)$ is given by $w(t)=1-\frac{1}{1+\chi e^{t}}$ where $\chi=\frac{\xi_{0}}{1-\xi_{0}}$. The function $w$ goes to 1 when $t$ goes to infinity. The functions $\xi$ and $w$ are equal in $\left(0, t_{1}\right)$ where $t_{1}=t_{r}+t_{*}$ and $t_{*}$ the first time such that $w(t)=\xi^{*}$ (this time exists because $w$ is a continuous function, starts at $\xi_{0}$ lower than $\xi^{*}$ and goes to 1$)$,

$$
t_{1}=\inf \{t>0, \xi(t-\tau)+\kappa \xi(t)>1-\Delta+\kappa\} .
$$

We can explicitly compute $t_{*}$ and $t_{1}$,

$$
\begin{gathered}
t_{*}=\log \left(\frac{\xi^{*}}{\chi\left(1-\xi^{*}\right)}\right), \\
t_{1}=\log \left(\frac{\nu+\sqrt{\nu^{2}+4 \xi^{*}\left(1-\xi^{*}\right) e^{-\tau}}}{2 \chi\left(1-\xi^{*}\right) e^{-\tau}}\right)
\end{gathered}
$$

and $\nu=-\left(1-\xi^{*}\right)\left(1+e^{-\tau}\right)+\left(\frac{1+\kappa e^{-\tau}}{1+\kappa}\right)$. One has $t_{*}+\tau>$ $t_{1}>t_{*}$ and $\xi_{1}=\xi\left(t_{1}\right)=w\left(t_{1}\right)>\xi^{*}$. Let consider the equation

$$
\begin{gathered}
\left\{\begin{array}{c}
\dot{v}(t)=-v(t)(1-v(t)) \\
v\left(t_{1}\right)=\xi_{1}
\end{array}\right. \\
\xi(t)=v(t)=\frac{1}{1+\frac{e^{t}}{\chi e^{2 t_{1}}}} \text { on }\left(t_{1}, t^{\prime}{ }_{1}\right) \text { where } \\
t_{1}^{\prime}=\log \left(\chi e^{2 t_{1}} \frac{\alpha+\sqrt{\alpha^{2}+4\left(1-\xi^{*}\right) \xi^{*} e^{-\tau}}}{2 e^{-\tau} \xi^{*}}\right)
\end{gathered}
$$

and $\alpha=-\xi^{*}\left(1+e^{-\tau}\right)+\left(\frac{1+\kappa e^{-\tau}}{1+\kappa}\right) \cdot \xi\left(t_{1}^{\prime}\right)=v\left(t_{1}^{\prime}\right)$ is lower than $\xi^{*}$.

Remark 1. Suppose that that $\kappa<1$. For all $\tau>0$, and for all initial conditions $\xi(t)=\xi_{0} \in(0,1)$ on $(-\tau, 0)$, the solution of imitation equation oscillates around the ESS.

Proof. Same arguments as in proposition 3.

\section{NUMERICAL INVESTIGATION: IMPACT OF THE REGRET COST AND THE DE- LAY ON THE CONVERGENCE TO THE ESS}

Impact of the cost $\kappa$ The ESS depends on the parameter $\kappa$. When $\kappa$ is large, the ESS point is closed to $(1,0):$ the pure strategy transmit. Our first numerical experiment studies the convergence of these dynamics for the case of the unit growth parameter $K$ and a small delay $\tau=0.02$ as a function of $\kappa$ : we check the speed of convergence and the stability of the dynamics as a function of the cost $\kappa$. The state $2 / 3$ is a stationary point for these parameters, for which $2 / 3$ of the population choose to transmit. We took $\Delta=1 / 3$.

Figure 4 (resp. Figure 5) represents the trajectories of the population using the strategy $T$ in the imitation dynamic (resp. replicator dynamic) when the initial condition is $\xi(t)=0.02 \in(-\tau, 0)$.

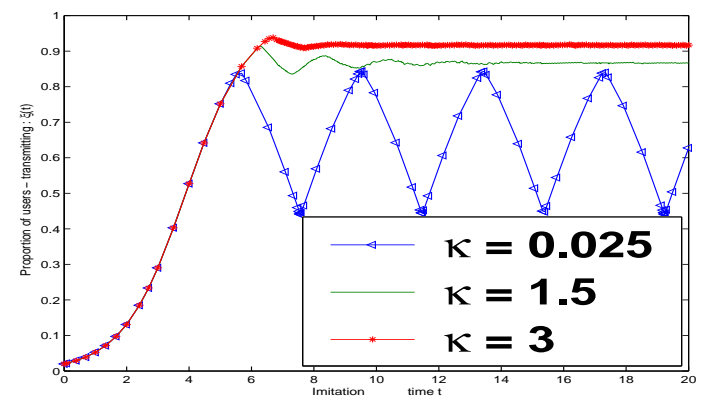

Figure 4: Effect of $\kappa$ on stability of imitation dynamic. $\tau=1$

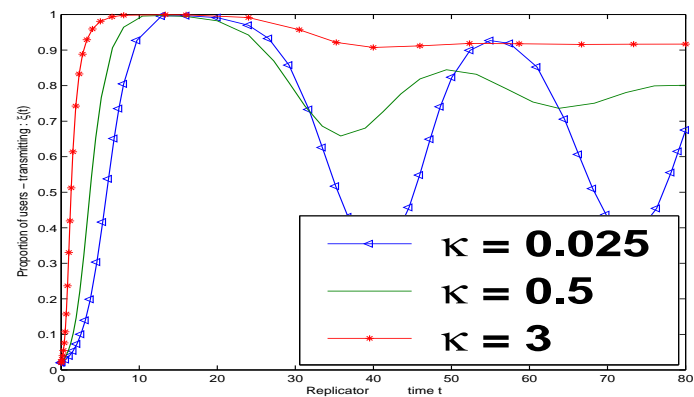

Figure 5: Effect of $\kappa$ on stability of replicator dynamic when $\tau=8$

Impact of the delay The ESS does not depends on the delay but has a big influence on the stability of the system. Our second numerical experiment studies the convergence of these dynamics for the case of the unit growth parameter $K$ as a function of delay: we check the speed of convergence 
and the stability of the dynamics as a function of the delay $\tau$. The state $2 / 3$ is a stationary point for these parameters, for which $2 / 3$ of the population choose to transmit. We took $\Delta=1 / 3, \kappa=0.002$. The resulting trajectories of the population using the strategy $T$ is represented as a function of time.

We evaluate the stability varying the delay $\tau$ between 0.02 and 15 time units in the replicator dynamic in figure 6 and figure 7. For $\tau=0.02$, we have stability but the convergence speed is slow. The other extreme is illustrated for $\tau=15$ which the trajectory oscillates rapidly and the amplitude is seen to be greater than $2 / 3$. The system is unstable.

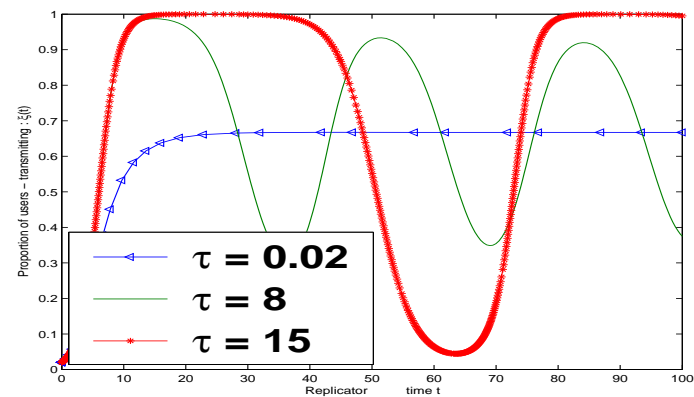

Figure 6: Effect of $\tau$ on stability of replicator dynamic when $\kappa=0.002$

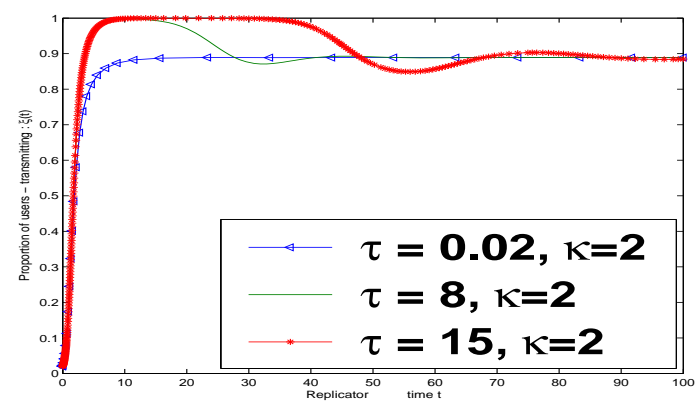

Figure 7: Replicator dynamic. $\kappa=2$. The system is always stable.

Figure 8 represents the trajectories of the population using the strategy $T$ in the imitation dynamic in which the system is unstable for all $\tau>0$. The amplitude of oscillation growth with the delay.

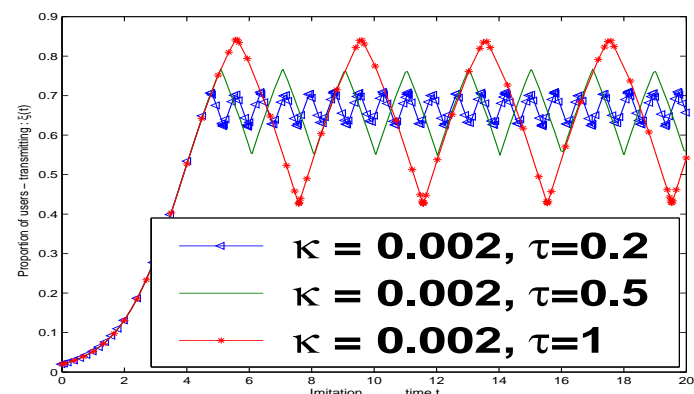

Figure 8: Imitation dynamic. Impact of $\tau$.

\section{CONCLUDING REMARKS}

The game studied in this paper, considers the population of nodes that have many local interactions between two nodes. Hence, the probability of success of a node is given by $\xi^{*}\left(1-\xi^{*}\right)$. It is easy to see that the probability of success is maximum when $\xi=1 / 2$. We can investigate the impact of regret cost on the equilibrium ESS and show how this cost can be used to enforce the population to converge ESS $\xi^{*}=1 / 2$.

Now, we can easily show that the population converge to the $\operatorname{ESS} \xi^{*}=1 / 2$ if the regret cost $\kappa^{*}=2 \Delta-1$ and $\Delta>1 / 2$ (see figure 9 ). When $\Delta<1 / 2$, the cost $\kappa^{*}=0$ gives best performances and the population converges to the $\operatorname{ESS} \xi^{*}=1-\Delta$.

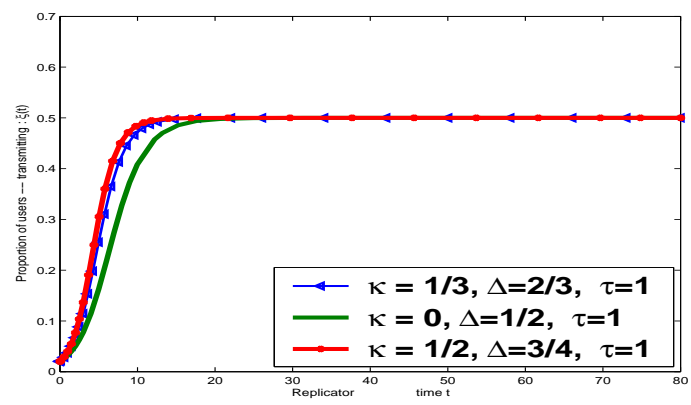

Figure 9: Replicator Dynamic. Optimal probability of success when $\kappa=1 / 3,0,1 / 2$.

\section{Acknowledgement}

This work was supported by the Bionets European Project.

\section{REFERENCES}

[1] E. Altman and T. Boulogne and R. Azouzi and T. Jimenez, A survey on networking games in telecommunications, Manuscript, 2000.

[2] Bellman R and Cooke K.L. Differential difference equations. Academic Press, New York, 1963.

[3] N. Bonneau, E. Altman, M. Debbah, and G. Caire. An Evolutionary Game Perspective to ALOHA with Power Control. 19th International Teletraffic Congress, Beijing, China, Aug. 29-Sep., 2005.

[4] T. Boulogne and E. Altman and H. Kameda and O. Pourtallier, Mixed equilibrium in multiclass routing games, IEEE Trans. on Automatic Control, 2001.

[5] R. Cressman. Evolutionary Dynamics and Extensive Form Games. MIT Press, Cambridge, 2003.

[6] J. Dieudonné, Foundations of Modern Analysis, Academic Press, New York, 1960.

[7] P. Dutta. A folk theorem for stochastic games. Journal of Economic Theory 66 : 1-32, 1995.

[8] Mark Felegyhazi and Jean-Pierre Hubaux. Game theory in wireless networks: A tutorial. EPFL Technical report: LCA-REPORT-2006-002, 2006.

[9] S. Fischer and B. Vöcking. On the evolution of selfish routing. In Proc. 12th Annural European Symposium on Algorithms (ESA), pages 323-334, 2004.

[10] Drew Fudenberg and David Levine. Efficiency and Observability with long-run and short-run players, 
volume 62. Journal of Economic Theory,p.103-135, 1994.

[11] Drew Fudenberg, David Levine, and Eric Maskin. The folk theorem with imperfect public information. Econometrica, Vol.62, Issue 5, p.997-1039, 1994.

[12] Drew Fudenberg, David K. Levine, and Satoru Takahashi. Perfect Public equilibrium when players are patient. Harvard, 2006.

[13] Drew Fudenberg and Eric Maskin. The Folk Theorem in repeated games with discounting or with incomplete information. Econometrica, Vol.54, No.3, pp.533-554., 1986.

[14] Drew Fudenberg and Eric Maskin. On the dispensability of public randomization in discounted repeated games. Journal of Economic Theory, Elsevier, vol. 53(2), pages 428-438, 1991.

[15] S. Ghez, S. Verd, and S. Schwartz, Stability properties of slotted-aloha with multipacket reception capability, IEEE Trans. Automatic Control, vol. 33, no. 7, 1988.

[16] Herbert Gintis. Game Theory Evolving. Princeton University Press, 2000.

[17] K. Gopalsamy. Stability and Oscillation in Delay Differential Equations of Population Dynamics. Kluwer Academic Publishers, London, 1992.

[18] J. Hofbauer and K. Sigmund. Evolutionary Games and Population Dynamics. Cambridge University Press, Cambridge, UK, 1998.

[19] J. Hofbauer and K. Sigmund. Evolutionary game dynamics. American Mathematical Society, Vol 40 No. 4, pp. 479-519, 2003.

[20] Y. Jin and G. Kesidis, Equilibria of a noncooperative game for heterogeneous users of an aloha network, IEEE communication Letters, vol.6, no. 7, 2002.

[21] A. MacKenzie and S. Wicker, Stability of Multipacket Slotted Aloha with Selfish Users and Perfect Information, in Proceedings of IEEE INFOCOM, 2003.

[22] A. MacKenzie and S. Wicker, Selfish users in Aloha: a game theoretic approach, in proceedings of the IEEE VTC, 2001.

[23] D. Mitra and Q. W. Wang, " Stochastic Traffic engineering for demand uncertain and risk-aware network", IEEE/ACM Transactions on networking, Vol. 13, No. 2, April, 2005.

[24] M. Lorenz, J. D. Gehrke and J. Hammer, "Situation-aware risk Management in autonomous Agents", CIKM'05, October, Bremen, Germany, 2005.

[25] R. Rom and M. Sidi, Multiple Access Protocols: Performance and Analysis, Springer-Verlag NY, 1990.

[26] T. Roughgarden and E. Tardos, How bad is selfish routing, in Proc. of the 41st Annual IEEE Symposium on the Foundations of Computer Science, 2000.

[27] L. Samuelson. Evolutionary Games and Equilibrium Selection. MIT Press, 1997.

[28] W.H. Sandholm. Potential games with continuous player sets. Journal of Economic Theory, 97:81-108,
2001.

[29] Shakkottai S., Altman E., and Kumar A. The case for non-cooperative multihoming of users to access points in ieee 802.11 wlans. IEEE Infocom, 2006.

[30] J Maynard Smith and GR Price. The logic of animal conflict, Nature 246, 15-18. 1973.

[31] Sorin S. On repeated games with Complete Information. Mathematics of Operations Research, 11, 147-160, 1986.

[32] H. Tembine, E. Altman and R. El-Azouzi. Delayed Evolutionary Game Dynamics applied to the Medium Access Control, to appear in Bionetworks 2007, available at http://www.lia.univavignon.fr/fich_art/934-dynamicsfinal.pdf

[33] H. Tembine and E. Altman. Asymmetric Delay in Evolutionary Games, to appear in ValueTools 2007, available at http://www.lia.univavignon.fr/fich_art/935-delay.pdf

[34] L. Teo, G. Ahn and Y. Zheng, "Dynamic and risk-aware network access management", Proc. of the eighth ACM symposium on Access control models and technologies, Como, Italy, 2003.

[35] T. L. Vincent and T. L. S. Vincent. Evolution and control system design. IEEE Control Systems Magazine, Vol 20 No. 5, pp 20-35, 2000.

[36] J.W. Weibull. Evolutionary Game Theory. Cambridge, MA: MIT Press,1995. 